\title{
ON THE FINITELY GENERATED SUBGROUPS OF AN AMALGAMATED PRODUCT OF TWO GROUPS
}

BY

\author{
R. G. BURNS
}

\section{In Memoriam Hanna Neumann}

\begin{abstract}
Sufficient conditions are found for the free product $G$ of two groups $A$ and $B$ with an amalgamated subgroup $U$ to have the properties (1) that the intersection of each pair of finitely generated subgroups of $G$ is again finitely generated, and (2) that every finitely generated subgroup containing a nontrivial subnormal subgroup of $G$ has finite index in $G$. The known results that Fuchsian groups and free products (under the obvious conditions on the factors) have properties (1) and (2) follow as instances of the main result.
\end{abstract}

1. Introduction. This paper extends the class of groups $G$ for which the following two properties are known to hold:

(1) every pair of finitely generated subgroups of $G$ intersect in a finitely generated subgroup (briefly, $G$ has the finitely generated intersection property);

(2) no finitely generated subgroup of $G$ of infinite index contains a nontrivial subnormal subgroup of $G$.

Our main result (Theorem 2.3) gives sufficient conditions for the generalized free product $(A * B ; U)$ of groups $A$ and $B$ amalgamating the subgroup $U$ to have properties (1) and (2). That some restriction is necessary is shown by the group $\left\langle x, y \mid x^{2}=y^{3}\right\rangle$, which has neither property (Karrass and Solitar [8], Moldavanskir [10]). The proof of Theorem 2.3 utilises the algebraic methods developed by Karrass and Solitar [8]. The following result is a particular case of that theorem. We call a subgroup $U$ of a group $A$ isolated if, whenever $a^{n} \in U$ for any nonzero integer $n$ and any $a \in A$, then $a \in U$.

1.1. Theorem. If $G=(A * B ; U)$ where $A$ is free and $U$ is infinite cyclic and isolated in $A$, then

(i) if $B$ bas the finitely generated intersection property, so does $G$;

(ii) if $U \neq A$, any finitely generated subgroup of $G$ containing a nontrivial subnormal subgroup of $G$ bas finite index.

Received by the editors July 15, 1971.

AMS 1970 subject classifications. Primary 20E30; Secondary 20E05, $20 \mathrm{E} 40$.

Key words and phrases. Amalgamated product, generalized free product, malnormal subgroup, subnormal subgroup, finitely generated intersection property, Kuroš rewriting process, Schreier system, compatible regular extended Schreier system, double ended coset. 
This implies in particular the result of Greenberg [3] that the Fuchsian groups (i.e. the discrete subgroups of $L F(2, R)$, the group of all $2 \times 2$ matrices over the reals with determinant +1 ) have properties (1) and (2). For, as Karrass and Solitar have pointed out, it is not difficult to show that a finite extension $G$ of a group with property (1) again has property (1), and that certainly if the nontrivial subnormal subgroups of $G$ are infinite, the same is true as regards property (2). Now the finitely generated infinite Fuchsian groups are either generalized free products of the sort described in Theorem 1.1 or finite extensions of such generalized free products, and their nontrivial subnormal subgroups are infinite: the first claim was originally proved by R. Fricke and F. Klein by geometrical methods, and recently by Hoare, Karrass and Solitar [5] using methods of combinatorial group theory; the second statement has recently been proved also by Hoare, Karrass and Solitar.( $\left.{ }^{1}\right)$

Our Theorem 2.3 is however of wider applicability. Thus it contains as a special case the result that a free product has property (1) if the factors do (Baumslag [1]), and has property (2) if there are at least two nontrivial factors (Karrass and Solitar [7]).

Property (1) was first considered by Howson [6], who established it for free groups. Property (2) originated with Schreier's well-known result that a finitely generated normal subgroup of a free group necessarily has finite index.

I am indebted to A. Karrass and D. Solitar for introducing me to the problem and for many helpful comments.

This research was partially supported by a grant from the National Research Council of Canada.

2. Statement of results. For concise statements of the results a few preliminaries are necessary.

For the usual definition, and existence and uniqueness, of a generalized free product see e.g. [9]. Alternatively one may define it by the following well-known characterizing property (which will be all that is used directly in the sequel). Recall first that a left transversal for a subgroup $H$ of a group $G$ is a complete set of representatives of left cosets $g H(g \in G)$.

2.1. Lemma (cf. [9, Theorem 4.4]). A group $G$ is a free product of two subgroups $A$ and $B$ amalgamating a subgroup $U$ contained in $A \cap B$ if and only if, for each pair $T_{A}, T_{B}$ of left transversals for $U$ in $A, B$ respectively, every element $g \in G$ can be uniquely expressed in the form

$$
g=t_{1} \cdots t_{n} u,
$$

where $n \geq 0, u \in U, t_{i} \in\left(T_{A} \cup T_{B}\right) \backslash U(i=1, \cdots, n)$ and $t_{i}, t_{i+1}$ do not both

(1) On subgroups of infinite index in Fuchsian groups, Math. Z. (to appear). 
belong to $T_{A}$ nor to $T_{B}(i=1, \cdots, n-1)$.

We write $G=(A * B ; U)$. We shall refer to the right-hand side of (3) as the canonical form for $g$ (relative to the pair $T_{A}, T_{B}$ ). The element $g$ will be said to be of length $n$, to begin with $t_{1}$ and to end with $t_{n}$. The $t_{i}$ will be called syllables ( $A$ - or $B$-syllables according as they belong to $T_{A}$ or $T_{B}$ ), and $u$ will be termed the $U$-syllable of $g$. Finally, the elements $t_{1} \cdots t_{i}(i=1, \cdots, n)$ will be called initial segments of $g$.

We shall also need the concept of malnormality. B. Baumslag [2] defined a subgroup $U$ of a group $G$ to be malnormal in $G$ if $g^{-1} U g \cap U$ is trivial for all $g \in G \backslash U$. A nontrivial example relevant to our purposes is that of an isolated cyclic subgroup of a free group (see $\$ 6$ ).

The following lemma furnishes an equivalent condition more convenient for us.

2.2. Lemma (Solitar). A subgroup $U$ is malnormal in $G$ if and only if there exists a left transversal $T$ for $U$ in $G$, containing the identity $e$, such that for all $t \in T \backslash\{e\}$ and all $u \in U, u t \in T \backslash\{e\}$; i.e. such that

$$
U(T \backslash\{e\})=T \backslash\{e\} .
$$

Proof. Suppose $U$ is malnormal in $G$ and let $T_{1}$ be a complete set of representatives of the double cosets $U g U(g \in G)$, including $e$. Then $T=U\left(T_{1} \backslash\{e\}\right)$ $U\{e\}$ is a left transversal for $U$ in $A$; for, if $t_{1}, t_{2} \in T_{1} \backslash\{e\}$ and $u_{1}, u_{2} \in U$ are such that $\left(u_{1} t_{1}\right)^{-1} u_{2} t_{2} \in U$, then $t_{1}^{-1} u_{1}^{-1} u_{2} t_{2} \in U$, whence $t_{1}=t_{2}$, and thence $u_{1}=u_{2}$ by the malnormality of $U$. Thus $T$ satisfies (4). The converse is equally straightforward and we omit the proof.

In addition to malnormality the following condition will be imposed. We shall say that a subgroup $U$ of $G$ is finitely involved in a subset $S \subseteq G$ with respect to a left transversal $T$ for $U$ in $G$, if there is a finite subset $V \subseteq U$ such that $S \subseteq$ $T V\left(S^{-1} S \cap U\right)$. We shall then say that $U$ is finitely involved throughout $G$ if there exists a left transversal $T$ for $U$ in $G$ such that, for every finitely generated subgroup $H \leq G$ and every $g \in G, U$ is finitely involved in $H g$, i.e. there is a finite subset $V$ of $U$, depending on $\mathrm{Hg}$, such that

$$
H g \subseteq T V\left(g^{-1} H g \cap U\right) \text {. }
$$

Clearly a finite subgroup of a group $G$ is finitely involved throughout $G$. However, for us the infinite case is more interesting: we shall prove in $\$ 6$ that any isolated infinite cycle in a free group $F$ is finitely involved throughout $F$. In fact by Theorem 6.1 such an infinite cycle satisfies the more stringent requirements of our main result, which we can now formulate.

2.3. Theorem. Let $G=(A * B ; U)$ where $U$ is malnormal in $A$ and finitely involved throughout $A$, and has the additional property that there is a left transversal 
$T$ for $U$ in A satisfying simultaneously conditions (4) and (5). The following conclusions are valid:

(i) if every subgroup of $U$ (including $U$ itself) is finitely generated, and $A$, $B$ bave the finitely generated intersection property, then so does $G$;

(ii) if $U \neq A, B$ and $H$ is a finitely generated subgroup of $G$ containing a subgroup $N, N \npreceq U, N$ subnormal in $G$, then $H$ bas finite index.

Theorem 1.1 follows at once from this theorem and Theorem 6.1.

3. Lemmas. The following lemma is crucial to the proof of Theorem 2.3. Broadly speaking it shows that the restriction given by the hypothesis of Theorem 2.3 on the way $U$ is embedded as a subgroup of a single factor of $G=(A * B ; U)$ implies a property similar to finite involvement of $U$ in certain subsets of the whole group $G$.

3.1. Lemma. Suppose that $G=(A * B ; U)$ where $U$ and $A$ satisfy the bypothesis of Theorem 2.3. Let $T_{A}$ be a left transversal for $U$ in $A$ satisfying (4) and (5), and let $T_{B}$ be any left transversal for $U$ in $B$, containing $e$. Let $H$ be any finitely generated subgroup of $G$ and let $g$ be any element of $G$. Then if $D$ denotes the set of all elements of $\mathrm{Hg}$ ending in an element of $T_{A} \backslash\{e\}$ and $D_{1}$ denotes the set obtained from $D$ by deleting the U-syllables from the ends of the elements of $D$, there exists a finite subset $V \subseteq U$ such that

$$
D \subseteq D_{1} V\left(g^{-1} H g \cap U\right) \text {. }
$$

For the proof we need some of the technique developed by Karrass and Solitar [8]. In order to elicit the structure of the subgroups of $(A * B ; U)$ they introduced the concept of a compatible regular extended Schreier system (or cress) for a subgroup. This has evolved from the idea of a Schreier transversal for a subgroup of a free group. In the case that $U$ is trivial the cress becomes the regular extended Schreier system employed in e.g. [9] to prove the Kuroš subgroup theorem for free products. We give a definition in terms of the canonical forms of elements. This gives the definition greater concreteness at considerable cost in generality: however it suffices for our purposes.

3.2. Definition (cf. Karrass and Solitar [8]). A compatible regular extended Schreier system (cress) for a subgroup $H$ of $G=(A * B ; U)$ relative to left transversals $T_{A}, T_{B}$ for $U$ in $A, B$ respectively, both containing $e$, is a pair $\left\{C_{A}, C_{B}\right\}$ of right transversals for $H$ in $G$, with the following properties:

(i) for all $g \in C_{A} \cup C_{B}$, where $g=t_{1} \cdots t_{n} u$ in canonical form,

(a) if $g \in C_{A}$, then $g u^{-1} \in C_{A}$ (and similarly for $C_{B}$ );

(b) if $u=e$ and $t_{n} \in T_{A}$, then $g, g t_{n}^{-1} \in C_{A}$ (and similarly, if $t_{n} \in T_{B}$, then 
$\left.g, g t_{n}^{-1} \in C_{B}\right)$;

(c) if $g u^{-1} \in C_{A} \cap C_{B}$, then $g \in C_{A} \cap C_{B}$;

(ii) if $S_{A}$ is the set consisting of $e$ and all $e \neq g \in C_{A}$ which have $u=e$ and $t_{n} \in T_{B}$, then $S_{A}$ is a complete double coset representative system for $G$ modulo $(H, A)$ (and similarly for $S_{B}$ );

(iii) if $R_{A}$ is the set of all $g \in C_{A}$ which have $u=e$, then $R_{A}$ is a complete double coset representative system for $G$ modulo $(H, U)$ (and similarly for $R_{B}$ ).

We remark that this fulfills the conditions defining a cress in $[8, \$ 5]$. To see this let all nontrivial elements of $U$ be, in the terminology of [8], generating symbols of $U$, and let the nontrivial elements of $T_{A} \cup U, T_{B} \cup U$ be the generating symbols of $A, B$ respectively. Conditions (1) to (4) of $[8, \$ 5]$ are then readily verified as being equivalent to the above. The proof of the existence of a cress as defined above is the same as that of [8, Lemma 6].

We now choose a particular generating set for $H$ in terms of a given cress $\left\{C_{A}, C_{B}\right\}$ for $H$ in $G$. For each $g \in G$ let $g \phi_{A}$ denote the representative in $C_{A}$ of $\mathrm{Hg}$, and define the coset representative function $\phi_{B}: G \rightarrow C_{B}$, analogously. For each $k \in C_{A}, x \in A$, write

$$
s_{A}(k, x)=k x\left((k x) \phi_{A}\right)^{-1},
$$

and define $s_{B}(k, x)$ for $k \in C_{B}, x \in B$, analogously. For each $d \in C_{B}$ define

$$
t(d)=d\left(d \phi_{A}\right)^{-1} \text {. }
$$

The Kuroš rewriting process for $H$ in terms of these $s$ - and $t$-symbols is then defined as follows. Suppose $b \in H$ has canonical form $t_{1} \cdots t_{n} u$, and write

$$
d_{i}^{A}=\left(t_{1} \cdots t_{i}\right) \phi_{A}, \quad d_{i}^{B}=\left(t_{1} \cdots t_{i}\right) \phi_{B} \quad(i=1, \ldots, n) .
$$

The rewritten expression for $b$ (cf. [9, p. 230]) is obtained by replacing $t_{i}$ $(i=1, \cdots, n-1)$ by

$$
t\left(d_{i-1}^{B}\right) \cdot s_{A}\left(d_{i-1}^{A}, t_{i}\right) \cdot t\left(d_{i}^{B}\right)^{-1} \text { if } t_{i} \in T_{A}
$$

and by

$$
s_{B}\left(d_{i-1}^{B}, t_{i}\right) \text { if } t_{i} \in T_{B}
$$

and replacing $t_{n} u$ by

$$
t\left(d_{n-1}^{B}\right) \cdot s\left(d_{n}^{A}, t_{n} u\right) \quad \text { if } t_{n} \in T_{A}
$$

and by

$$
s\left(d_{n}^{B}, t_{n} u\right) \text { if } t_{n} \in T_{B} .
$$


It is not difficult to verify that these replacements leave $b$ unchanged. Notice also that if $d \in C_{B}$ has the form $r u_{1}$ where $r \in R_{B_{*}}, u_{1} \in U$, then (cf. [8, p. 243])

$$
t(d)=r u_{1}\left(\left(r u_{1}\right) \phi_{A}\right)^{-1}=r\left(r \phi_{A}\right)^{-1} \cdot\left(r \phi_{A} u_{1}\left(\left(r u_{1}\right) \phi_{A}\right)^{-1}\right)
$$

that is

$$
t(d)=t(r) \cdot s_{A}\left(r \phi_{A}, u_{1}\right)
$$

It follows that $H$ is generated by the $t(r)\left(r \in R_{B}\right)$ and the $s$-symbols. Consider the element $s_{A}(k, x)$, where $x \in A$, and $k \in C_{A}$ has the form $d q u$, where $d \in S_{A}$, $q \in T_{A}$ (and $d q \in R_{A}$ ) and $u \in U$. It follows from (ii) of Definition 3.2 that $(d q u x) \phi_{A}=d q_{1} u_{1}$ where $q_{1} \in T_{A}, u_{1} \in U$. Thus

$$
s_{A}(k, x)=d\left(q u x u_{1}^{-1} q_{1}^{-1}\right) d^{-1} \in d A d^{-1} \cap H .
$$

Similarly, when $k \in C_{B}$ and $x \in B$, we have $s_{B}(k, x) \in d_{1} B d_{1}^{-1} \cap H$ where $d_{1} \epsilon$ $S_{B}$ is the representative of the double coset $H k B$. Write

$$
\begin{aligned}
R & =\left\{t(r) \mid r \in R_{B}\right\}, \\
Q_{A} & =\left\{d \mid d \in S_{A^{\prime}}, d A d^{-1} \cap H \nless d U d^{-1}\right\}, \\
Q_{B} & =\left\{d \mid d \in S_{B^{\prime}} d B d^{-1} \cap H \ddagger d U d^{-1}\right\} .
\end{aligned}
$$

The above facts are summarised and supplemented in the following lemma.

3.3. Lemma. Let $H \leq G=(A * B ; U)$ and let $\left\{C_{A}, C_{B}\right\}$ be a cress for $H$ relative to $T_{A}, T_{B}$. Then $H$ is generated by the set $R$ together with $H \cap U$ and all subgroups $d A d^{-1} \cap H\left(d \in Q_{A}\right)$ and $d B d^{-1} \cap H\left(d \in Q_{B}\right)$. Furthermore, if every subgroup of $U$ (including $U$ itself) is finitely generated, then $H$ is finitely generated if and only if $R, Q_{A}$ and $Q_{B}$ are finite, and for all $g \in G, g A g^{-1} \cap H$ is finitely generated.

The proof of the first part of this lemma is sketched above (see also [8, Lemma 7]). The second part is immediate from Lemma 3 and Theorems 4, 5 of [8].

Write $R_{1}=\left\{r, r \phi_{A} \mid r \in R_{B}, t(r) \neq e\right\}$, and write $P$ for the set of all $A$-syllables of elements of the set $R_{1} \cup Q_{A} \cup Q_{B}$.

3.4. Corollary. The subgroup $H \leq(A * B ; U)$ is finitely generated only if $P$ is finite.

3.5. Lemma. Let $H \leq G=(A * B ; U)$ and let $\left\{C_{A}, C_{B}\right\}$ be a cress for $H$ relative to $T_{A}, T_{B}$. Suppose that $g \in R_{A}$ and $b \in H$ are such that, in canonical form, $b g=t_{1} \cdots t_{n} u \notin U$, where $e \neq t_{n} \in T_{A}$. Then $t_{n} u=u_{1} q_{1}^{-1} a q_{2}$, where $u_{1} \in U, q_{1} \epsilon$ $P \cup\{e\}$, either $q_{2}=e$ or $q_{2}$ is the last syllable of $g$, and $a \in A \cap d^{-1} H d$ where $d q_{2}=g$. 
Proof. Firstly we apply to $h g=t_{1} \cdots t_{n} u$ a rewriting process similar to the Kuroš rewriting process described above. To be specific, we replace $t_{i}$ $(i=1, \cdots, n-1)$ by the appropriate one of the products (7), (8), but $t_{n} u$ is replaced by $t\left(d_{n-1}^{B}\right) \cdot s_{A}\left(d_{n-1}^{A}, t_{n} u\right) \cdot g$. This process does not alter $b g:$ for example

$$
\begin{aligned}
& t\left(d_{n-1}^{B}\right) \cdot s_{A}\left(d_{n-1}^{A}, t_{n} u\right) \cdot g \\
& \quad=d_{n-1}^{B}\left(d_{n-1}^{A}\right)^{-1} d_{n-1}^{A} t_{n} u\left(\left(d_{n-1}^{A} t_{n} u\right) \phi_{A}\right)^{-1} \cdot g=d_{n-1}^{B} \cdot{ }_{n} u,
\end{aligned}
$$

since $\left(d_{n-1}^{A}{ }_{n} u\right) \phi_{A}=(b g) \phi_{A}=g$; and then $d_{n-1}^{B}$ cancels with part of the element (8) replacing $t_{n-1}$, and so on.

Write $d_{n-1}^{A}=d q_{1} u_{1}$ where $d \in S_{A}$ is the representative of the double coset $H d_{n-1}^{A} A, q_{1} \in T_{A}$ and $u_{1} \in U$. Then by Definition 3.2(ii), $g=d q_{2}$ where $q_{2} \epsilon$ $T_{A}$, since $H g A=H d_{n-1}^{A} A$. From (10) we have

$$
t_{n} u=\left(d_{n \rightarrow 1}^{A}\right)^{-1} \cdot d_{n-1}^{A} t_{n} u g^{-1} \cdot g=u_{1}^{-1} q_{1}^{-1} d^{-1} \cdot d\left(q_{1} u_{1} t_{n} u q_{2}^{-1}\right) d^{-1} \cdot d q_{2} .
$$

Write $a=q_{1} u_{1} t_{n} u q_{2}^{-1}$. Then $a \in A \cap d^{-1} H d$ and $t_{n} u=u_{1}^{-1} q_{1}^{-1} a q_{2}$. It remains to show only that if $q_{1} \neq e$ then $q_{1} \in P$.

Thus suppose $q_{1} \neq e$. If $d_{n-1}^{B}=d_{1} p_{1} v_{1} \neq d_{n-1}^{A}=d q_{1} u_{1}$, where $d_{1} \in S_{B}$, $p_{1} \in T_{B}, v_{1} \in U$, then by Definition 3.2(iii) $\left(d_{1} p_{1}\right) \phi_{A}=d q_{1} w_{1}$ where $w_{1} \in U$ and $d_{1} p_{1} \neq d q_{1} w_{1}$. Then we should have $d q_{1} w_{1} \in R_{1}$, whence $q_{1} \in P$. Thus we may assume that $d_{n-1}^{B}=d_{n-1}^{A}$. We next prove that $n>2$. If $n=1$ then $d_{n-1}^{A}=e$, which is impossible since $d_{n-1}^{A}$ has $q_{1}$ as its last syllable. If $n=2$ then $t_{1} \epsilon$ $T_{B}$ and $d_{n-1}^{B}=d q_{1} u_{1}^{-1}=d_{1}^{B}=t_{1} \phi_{B}$. Thus $H d q_{1} B=H t_{1} B=H B$, whence, by 3.2 , $d q_{1}=e$, which is impossible since $q_{1} \not t e$.

For all odd $j$ such that $1 \leq j \leq n-1$ write $d_{n-j}^{A}=d_{j} q_{j} u_{j}$ where $d_{j} \in S_{A}, q_{j}$ $\in T_{A}$ and $u_{j} \in U$; for $j$ even $(1 \leq j \leq n-1)$ write $d_{n-j}^{B}=d_{j} q_{j} u_{j}$ where $d_{j} \in S_{B}$, $q_{j} \in T_{B}$ and $u_{j} \in U$. Note that $t_{n-j} \in T_{B}$ or $T_{A}$ according as $j$ is odd or even. We shall prove that if $j$ is odd $(1 \leq j \leq n-2)$ and $d_{n-j}^{B}=d_{n-j}^{A}=d_{j} q_{j} u_{j}$ where $q_{j}$ $\neq e$, then either $d_{j} q_{j}$ is an initial segment of an element of $R_{1} \cup Q_{A} \cup Q_{B}$, or $d_{n-j-1}^{A}=d_{n-j-1}^{B}=d_{j} q_{j} q_{j+1} u_{j+1}$ where $e \neq q_{j+1} \in T_{B}$; and that if $j$ is even $(1 \leq j \leq n-2)$ the same statement is true except that $e \neq q_{j+1} \in T_{A}$.

Thus suppose $j$ is odd $(1 \leq j \leq n-2), e \neq q_{j} \in T_{A}, d_{j} q_{j}$ is not an initial segment of any element of $R_{1} \cup Q_{A} \cup Q_{B}$, and $d_{n-j}^{B}=d_{n-j}^{A}$. By Definition 3.2 (ii), since $d_{n-j}^{B}=d_{n-j}^{A}=d_{j} q_{j} u_{j}$, we must have $d_{n-j-1}^{B}=d_{j} q_{j} p_{j+1} v_{j+1}$, where $p_{j+1} \in T_{B}$ and $v_{j+1} \in U$. Then

$$
\begin{aligned}
s_{B}\left(d_{n-j-1}^{B}, t_{n-j}\right) & =d_{j} q_{j}\left(p_{j+1} v_{j+1} t_{n-j} u_{j}^{-1}\right)\left(d_{j} q_{j}\right)^{-1} \\
& =d_{j} q_{j} b\left(d_{j} q_{j}\right)^{-1} \text {, say. }
\end{aligned}
$$


Since $t_{n-j} \notin U$ it follows that $b \notin U$ if $p_{j+1}=e$. But then $d_{j} q_{j} \in Q_{B}$. We must therefore have $p_{j+1} \neq e$. Next suppose that $d_{n-j-1}^{A} \neq d_{n-j-1}^{B}$. Again by Definition 3.2 (ii) this implies that $d_{j} q_{j} p_{j+1} \neq\left(d_{j} q_{j} p_{j+1}\right) \phi_{A}$ whence $d_{j} q_{j}$ is an initial segment of an element of $R_{1}$. Therefore $d_{j} q_{j} p_{j+1}=d_{j+1} q_{j+1}$ (i.e. $p_{j+1}=q_{j+1}$ and $\left.d_{j} q_{j}=d_{j+1}\right)$, and $d_{n-j-1}^{A}=d_{j+1} q_{j+1} u_{j+1}$, where $e \neq q_{j+1} \in T_{B}$. A similar argument disposes of the case $j$ even.

We now apply this to complete the proof. We have by assumption that $d_{n-1}^{B}=$ $d_{1} q_{1} u_{1}$ where $e \neq q_{1} \in T_{A}$. By what we have just proved, if $d_{1} q_{1}$ were not an initial segment of any element of $R_{1} \cup Q_{A} \cup Q_{B}$, we should have $d_{n-2}^{B}=d_{n-2}^{A}=$ $d_{1} q_{1} q_{2} u_{2}$ where $e \neq q_{2} \in T_{B}$; and then since $d_{1} q_{1} q_{2}$ cannot be such an initial segment, it would follow that $d_{n-3}^{B}=d_{n-3}^{A}=d_{1} q_{1} q_{2} q_{3} u_{3}$, where $e \neq q_{3} \in T_{A}$, and so on. We should finally arrive at the situation where $d_{1}^{B}=d_{1}^{A}=d_{1} q_{1} \cdots q_{n-1} u_{n-1}$. This is impossible since if $t_{1} \phi_{A}=t_{1} \phi_{B}$, then by 3.2 (ii), this element can have length at most 1 , whereas $d_{1} q_{1} \cdots q_{n-1} u_{n-1}$ has length at least 2 . This completes the proof of the lemma.

Proof of Lemma 3.1. Suppose that $g_{1} \in H g \backslash U$ has the canonical form $g_{1}=$ $t_{1} \cdots t_{n} u$, where $t_{n} \in T_{A}$, and that we have a cress for $H$ in $G$, relative to $T_{A}$, $T_{B}$. Let $g_{2} \in R_{A}$ be the representative of the double coset $H g U$. Then $g=$ $b g_{2} u_{1}$ where $b \in H$ and $u_{1} \in U$. By Lemma 3.5,

$$
t_{n} u=u_{2} q_{1}^{-1} a q_{2} u_{1}=u_{2} q_{1}^{-1} a q_{1}\left(q_{1}^{-1} q_{2} u_{1}\right) \text {, }
$$

where $u_{2} \in U, q_{1} \in P \cup\{e\}$, either $q_{2}=e$ or $q_{2}$ is the final syllable of $g_{2}$, and $a \in A \cap d^{-1} H d$ where $g_{2}=d q_{2}$.

The element $q_{1}^{-1} a q_{1}\left(q_{1}^{-1} q_{2} u_{1}\right)$ lies in the coset $\left(A \cap\left(d q_{1}\right)^{-1} H d q_{1}\right)\left(q_{1}^{-1} q_{2} u_{1}\right)$. Since $H$ is finitely generated, by Lemma 3.3 so is $A \cap\left(d q_{1}\right)^{-1} H d q_{1}$. Therefore since the transversal $T_{A}$ satisfies condition (5) of the definition of finite involvement, there exists a finite subset $V \subseteq U$ such that

$$
\left(A \cap\left(d q_{1}\right)^{-1} H d q_{1}\right)\left(q_{1}^{-1} q_{2} u_{1}\right) \subseteq T_{A} V\left(g^{-1} H g \cap U\right) .
$$

(We have used here that $\left(q_{1}^{-1} q_{2} u_{1}\right)^{-1}\left(A \cap\left(d q_{1}\right)^{-1} H d q_{1}\right)\left(q_{1}^{-1} q_{2} u_{1}\right) \cap U=$ $\left.\left(g_{2} u_{1}\right)^{-1} H\left(g_{2} u_{1}\right) \cap U=g^{-1} H g \cap U.\right)$ It is clear from (12) that $V$ depends only on $g_{2}, q_{1}, q_{2}$ and $u_{1}$. However $g_{2}$ and $u_{1}$ are determined uniquely by $g$ (and if $q_{2} \neq e$, then it also is determined by $g$ ). It follows from (11) that for each $g_{1}=t_{1} \cdots t_{n} u \epsilon$ $H g \backslash U$, with $t_{n} \in T_{A} \backslash\{e\}$, we have

$$
{ }_{n} u \in u_{2} T_{A} V\left(g^{-1} H g \cap U\right),
$$

where $V \subseteq U$ is finite and depends only on $q_{1}, q_{2}$ and $g$. Now $P$ is finite by Corollary 3.4; hence since $q_{1} \in P \cup\{e\}$ and since there are only two possibilities for $q_{2}$, there are only finitely many pairs $q_{1}, q_{2}$. For each such pair let $V\left(q_{1}, q_{2}\right)$ $\subseteq U$ be a finite subset satisfying (12). Let $V_{1}$ be the union of the $V\left(q_{1}, q_{2}\right)$, 
taken over all pairs $q_{1}, q_{2}$. Then for all $g_{1}=t_{1} \cdots t_{n} u$ as above, we have

$$
t_{n} u \in U T_{A} V_{1}\left(g^{-1} H g \cap U\right) \text {. }
$$

However, since $T_{A}$ satisfies condition (4), and since $t_{n} \in T_{A} \backslash\{e\}$, it follows that $u \in V_{1}\left(g^{-1} H g \cap U\right)$, from which the desired inclusion (6) follows.

We require one final result. Let $G=(A * B ; U)$ and let $T_{A}, T_{B}$ be left transversals for $U$ in $A, B$ respectively. A subset $X \subseteq G$ is called double ended if it contains at least one element with its ending in $T_{A}$ and at least one element with its ending in $T_{B}$.

3.6. Lemma [8, Lemma 8]. Let $H \leq G=(A * B ; U)$ and suppose $Q_{A}, Q_{B}$ and $R_{1}$ are defined as above in terms of a cress for $H$ in $G$. Then $Q_{A} \cup Q_{B} \cup R_{1}$ is finite if and only if the number of double ended $(H, U)$ cosets in $G$ is finite.

4. The finitely generated intersection property. Theorem 2.3 (i) is an immediate consequence of Lemmas 3.3, 3.6 and the following result.

4.1. Lemma. Let $G=(A * B ; U)$ where $U$ and $A$ satisfy the bypothesis of Theorem 2.3, let $T_{A}$ be a left transversal for $U$ in $A$ satisfying (4) and (5) and let $T_{B}$ be any left transversal for $U$ in $B$, containing e. If $H$ and $K$ are finitely generated subgroups of $G$, then each intersection of an $(H, U)$ coset with a $(K, U)$ coset contains only finitely many $(H \cap K, U)$ cosets containing elements ending in elements of $T_{A} \backslash\{e\}$ (and therefore contains only finitely many double ended $(H \cap K, U)$ cosets $)$.

Proof. Suppose on the contrary that $g_{1}, g_{2} \in G$ are such that $H g_{1} U \cap K g_{2} U$ contains infinitely many $(H \cap K, U)$ cosets containing elements with endings in $T_{A}$. Let $Y=\left\{y_{1}, y_{2}, \ldots\right\}$ be a set of representatives of a countably infinite set of distinct $(H \cap K, U)$ cosets such that, for all $i, y_{i}$ ends in an element of $T_{A} \backslash\{e\}$. We may also assume that, for all $i, y_{i}$ has $U$-syllable $e$ since $y_{i}$ and $y_{i} u$ represent the same $(H \cap K, U)$ coset for all $u \in U$. Write

$$
y_{i}=b_{i} g_{1} u_{i}=k_{i} g_{2} v_{i} \quad(i=1,2, \ldots),
$$

where $b_{i} \in H, k_{i} \in K, u_{i}, v_{i} \in U$. Let $W_{H}$ and $W_{K}$ be left transversals in $U$ for $g_{1}^{-1} H g_{1} \cap U$ and $g_{2}^{-1} H_{2} \cap U$ respectively. Then we may assume that $u_{i}^{-1} \in W_{H}$ and $v_{i}^{-1} \in W_{K}$, for all $i$. For, suppose for instance that $u_{i}^{-1}=w_{i} u_{i}^{\prime}$ where $u_{i}^{\prime} \epsilon$ $g_{1}^{-1} H g_{1} \cap U$ and $w_{i} \in W_{H}$. Then $u_{i}=\left(u_{i}^{\prime}\right)^{-1} w_{i}^{-1}$, and

$$
b_{i} g_{1} u_{i}=b_{i} g_{1}\left(u_{i}^{\prime}\right)^{-1} g_{1}^{-1} \cdot g_{1} w_{i}^{-1}=b_{i}^{\prime} g_{1} w_{i}^{-1} \text {, }
$$

where $b_{i}^{\prime} \in H$.

Thus $b_{i} g_{1}=y_{i} u_{i}^{-1}$ and $k_{i} g_{2}=y_{i} v_{i}^{-1}$, where $u_{i}^{-1} \in W_{H}$ and $v_{i}^{-1} \in W_{K}$. By 
virtue of Lemma 3.1 all but finitely many of the $u_{i}$ are equal, and the same is true for the $v_{i}$. Hence there exists a pair $j, l, j \neq l$, of positive integers, such that $u_{j}=u_{l}$ and $v_{j}=v_{l}$. But then

$$
y_{j} y_{l}^{-1}=b_{j} b_{l}^{-1}=k_{j} k_{l}^{-1} \in H \cap K
$$

which contradicts our choice of $y_{j}, y_{l}$ as representatives of distinct $(H \cap K, U)$ cosets. This completes the proof.

Remark. An entirely similar proof shows that finite involvement of a subgroup $U$ throughout a group $A$ implies that for every pair of finitely generated subgroups $H, K$ of $A, H a_{1} U \cap K a_{2} U$ contains only finitely many $(H \cap K, U)$ cosets, for all $a_{1}, a_{2} \in A$. I have been unable to decide whether or not, conversely, the latter condition implies that $U$ is finitely involved throughout $A$.

5. Subnormal subgroups. In this section we shall prove Theorem 2.3 (ii). For this we require the following lemma (cf. [8, Theorem 10]).

5.1. Lemma. Let $G=(A * B ; U)$ where $U \neq A, U \neq B$, and let $H$ be a finitely generated subgroup containing a subnormal subgroup $N$ of $G, N \npreceq U$. Then $H$ bas finite index in $G$ if and only if the intersection of each conjugate of $H$ with $U$ bas finite index in $U$.

The idea of the proof is the same as that of [8, Theorem 10] and [1, p. 679]. For convenience we formulate and prove as a separate lemma a result used in the proof.

5.2. Lemma. Let $G=(A * B ; U)$ and let $T_{A}, T_{B}$ be left transversals, containing $e$, for $U$ in $A, B$. Suppose that $U$ bas index $>2$ in $A$ and $U \neq B$. Then every subnormal subgroup $N$ of $G, N \npreceq U$, contains two elements whose initial and terminal syllables lie in $T_{A} \backslash\{e\}$, with distinct initial syllables, and a third element which begins and ends in elements of $T_{B} \backslash\{e\}$.

Proof. It clearly suffices to show that whenever $N \triangleq K<G$ where $K$ contains such a triple of elements and $N \npreceq U$, then $N$ also contains such a triple. This is proved as follows. Suppose that $g_{1}, g_{2} \in K$ have distinct initial syllables and both begin and end with elements of $T_{A} \backslash\{e\}$, and that $g_{3} \in K$ begins and ends in elements of $T_{B} \backslash\{e\}$. Let $x \in N \backslash U$.

Suppose first that $x$ begins and ends in elements of $T_{B} \backslash\{e\}$. Then $g_{1} x g_{1}^{-1}$ and $g_{2} \times g_{2}^{-1}$ both belong to $N$, end and begin in elements of $T_{A} \backslash\{e\}$, and have distinct initial syllables since these are the same as those of $g_{1}$ and $g_{2}$ respectively. Then the three elements $g_{1} x g_{1}^{-1}, g_{2} x g_{2}^{-1}$, and $x$ have the required properties.

Second, suppose that $x$ begins in an element of $T_{A} \backslash\{e\}$ and ends in an element of $T_{B} \backslash\{e\}$. Since $g_{1}$ and $g_{2}$ have distinct initial syllables, at least one of 
the se syllables is different from the initial syllable of $x$ : suppose without loss of generality that $g_{1}$ has this property; then $g_{1}^{-1} x g_{1}$ belongs to $N$ and both begins and ends in elements of $T_{A} \backslash\{e\}$. Hence the element $g_{3}^{-1} g_{1}^{-1} \times g_{1} g_{3}$ belongs to $N$ and begins and ends in elements of $T_{B} \backslash\{e\}$. This is the case first dealt with.

If $x$ begins and ends in elements of $T_{A} \backslash\{e\}$ then $g_{3}^{-1} x g_{3}$ begins and ends in elements of $T_{B} \backslash\{e\}$ and again we are in the first case. Finally, if $x$ begins with an element of $T_{B} \backslash\{e\}$ and ends with an element of $T_{A} \backslash\{e\}$, consider instead $x^{-1}$ which falls into the second case above.

Proof of Lemma 5.1. Assume that $U$ has index $>2$ in $A$ : The contrary case is, with minor modification, treated as in [8, Theorem 10$]$.

Let

$$
N=N_{0} \Delta N_{1} \Delta \cdots \Delta N_{l}=G \quad(l \geq 0)
$$

be a shortest subnormal chain connecting $N$ and $G$. We first show that for all $g \in G \backslash U$ there exists an element $x \in G \backslash U$ such that (i) $x$ begins and ends in syllables of type different from that of the final syllable of $g$ (i.e. if $g$ ends in an $A$-syllable then $x$ begins and ends in B-syllables, and if $g$ ends in a $B$-syllable then $x$ begins and ends in $A$-syllables) and (ii) $\mathrm{gxg}^{-1} \in N$.

This is trivially so if $l=0$ (i.e. if $N=G$ ). Suppose $l>0$ and, as inductive hypothesis, that for each $g \in G \backslash U$ and each subnormal subgroup possessing a shorter subnormal chain (and not contained in $U$ ) there exists an element $x$ satisfying (i) and (ii) above. Let $x$ be such an element for $g$ and $N_{1}$. Then $g x g^{-1} \in N_{1}$. By Lemma 5.2 there is an element $g_{1} \in N$ which begins and ends in syllables of type different from that of the initial syllable of $g$. Then $g x^{-1} g^{-1} g_{1} g x g^{-1} \in N$, and $x_{1}=x^{-1} g^{-1} g_{1} g x$ begins and ends in syllables different in type from the final syllable of $g$; also $g x_{1} g^{-1} \in N$. This completes the inductive step.

Suppose $g \in G \backslash U$ and let $x \in G \backslash U$ be an element satisfying (i) and (ii) above. Then the double coset $\mathrm{HgU}$ can be written $\mathrm{Hgxg}^{-1} \mathrm{gU}=\mathrm{HgxU}$, since $H \geq N$. Hence every $(H, U)$ coset (including $H U$, by Lemma 5.2) is double ended. Since $H$ is finitely generated we infer from Lemma 3.6 that there are only finitely many $(H, U)$ cosets in $H$. However since $\left(U: g^{-1} H g \cap U\right)$ is finite for all $g \in G$ by hypothesis, this implies that $H$ has finite index in $G$. The remark that the "only if" part of 5.1 is trivial completes the proof.

Theorem 2.3(ii) follows immediately from Lemma 5.1 and the following result.

5.3. Lemma. Let $G=(A * B ; U)$ where $U(\neq A, B)$ and $A$ satisfy the bypothesis of Theorem 2.3, let $T_{A}$ be a left transversal for $U$ in $A$ satisfying (4) and (5), and let $T_{B}$ be any left transversal for $U$ in $B$, containing $e$. Let $H$ be a finitely generated subgroup containing a subnormal subgroup $N$ of $G, N \npreceq U$. Then the intersection of $U$ with each conjugate of $H$ bas finite index in $U$. 
Proof. Suppose that for some $g \in G, g^{-1} H g \cap U$ has infinite index in $U$. We may suppose $g=e$ by replacing $H$ by $g^{-1} H g$ and $N$ by $g^{-1} N g$. Let $W$ be a left transversal for $H \cap U$ in $U$.

We shall show that every subnormal subgroup $N, N \npreceq U$, has the property that every element of $W$ occurs as the representative of the $U$-syllable of some element of $N \backslash U$, beginning and ending in $A$-syllables.

This is trivially true for $G$ itself. Suppose that

$$
N=N_{0} \Delta N_{1} \Delta \ldots \Delta N_{l}=G \quad(l>0)
$$

is a shortest subnormal chain beginning with $N$ and, as inductive hypothesis, that the aforementioned property is possessed by subnormal subgroups with shorter subnormal chains. Thus $N_{1}$ is assumed to have the property. Suppose $g_{1} \epsilon$ $N_{1} \backslash U$ begins and ends in $A$-syllables, and has $U$-syllable $u$. By Lemma 5.2 there is an element $g_{2} \in N \backslash U$ that ends and begins with $B$-syllables. Then $g_{1}^{-1} g_{2} g_{1} \epsilon$ $N$ has $U$-syllable $u$ since $T_{A}$ satisfies condition (4), and both ends and begins with $A$-syllables. Thus $N$ (and hence $H$ ) has the property that every element of $W$ occurs as the representative of the $U$-syllable of some element of $N$ ending in an $A$-syllable. This contradicts (6) of Lemma 3.1, and the proof is complete.

6. Isolated cyclic subgroups of free groups. Theorem 1.1 is a special case of Theorem 2.3, established above. We have only to show that an isolated infinite cyclic subgroup of a free group satisfies the hypothesis of Theorem 2.3.

6.1. Theorem. Let $F$ be a free group freely generated by a set $X$ and let $U$ be an isolated nontrivial cyclic subgroup of $F$. Then $U$ is malnormal in F. Further there is a left transversal $T$ for $U$ in $F$ satisfying (4), such that for any element $g$ of $F$ there exists a finite subset $V \subset U$ such that

$$
H g \subseteq T V\left(g^{-1} H g \cap U\right) ;
$$

i.e. $U$ is finitely involved throughout $F$ with respect to $T$.

Proof. It is a simple exercise to show that $U$ is malnormal in $F$ and we omit its proof.

We may assume that a generator $u$ of $U$ is cyclically reduced; for if not then some conjugate $f^{-1} u f$ is, and then we may consider $f X f^{-1}$ in place of $X$.

Choose $T$ as follows. Let $T_{1}$ be a complete set of representatives for cosets $U f U(f \in F)$ such that each representative is an element of smallest (reduced) length in its double coset. Then set $T=U\left(T_{1} \backslash\{e\}\right) \cup\{e\}$. By the proof of Lemma 2.2, $T$ is a left transversal for $U$ in $F$.

If $g^{-1} \mathrm{Hg} \cap U \neq\{e\}$, then it has finite index in $U$ and (13) follows trivially. Assume therefore that $g^{-1} H g \cap U=\{e\}$.

Let $t$ be any element of $T_{1} \backslash\{e\}$. Then the meets (i.e. largest common initial 
segments) of $u$ with $t$ and $t^{-1}$ have lengths at most half the length of $u$, by the choice of $t$ as a shortest element of $U t U$. It follows that, if $l, k$ are nonzero integers of signs $\epsilon, \delta(= \pm 1)$ respectively and $v$ is the reduced form of $u^{\epsilon} t u^{\delta}$, then $w=u^{l-\epsilon} v u^{k-\delta}$ is reduced as written, i.e. no cancellation occurs.

Now suppose that (13) does not hold. By the choice of $T$ this means that there is an infinite set $\left\{w_{1}, w_{2}, \ldots\right\} \subseteq H g$ such that, in reduced form,

$$
w_{i}=u^{l_{i}} v_{i} u^{-k_{i}} \quad(i=1,2, \ldots)
$$

where (by replacing $u$ by $u^{-1}$ if necessary) $k_{i}$ is a positive integer, $k_{i} \rightarrow \infty$, and $l_{i}$ is an integer. Since $w_{i} \in H g$, we have $g w_{i}^{-1} \in H(i=1,2, \ldots)$. Hence there exists a positive integer $n$ such that, for all $i>n, g u^{k_{i}}$ is an initial segment of an element of $H$. For $i>n$ write $g u^{k_{i}}=g_{1} u^{n_{i-n}}$ in reduced form, where $n_{j} \geq 0$ $(j=1,2, \ldots)$ and $n_{j} \rightarrow \infty$ as $j \rightarrow \infty$.

Let $S$ be a right Schreier transversal for $H$ in $F$ (i.e. a complete set of right coset representatives, containing $e$, and closed under taking initial segments). Let $a_{1}, \cdots, a_{r}$ be Schreier free generators of $H$ constructed from $S$ and $X$ (i.e. the nontrivial elements of the form $s x((s x) \phi)^{-1}$, where $s \in S, x \in X$ and $(s x) \phi$ is the representative in $S$ of $H s x)$. For each $j=1,2, \cdots$, let $b_{j} \in H$ be a shortest element of $H$ in the generators $a_{1}, \cdots, a_{r}$, such that $g_{1} u^{n_{j}}$ is an initial segment of $b_{j}$. Suppose $b_{j}$ ends in $a_{m(j)}^{\delta(j)}$ where $\delta(j)= \pm 1$, and write

$$
b_{j}^{\prime} a_{m(j)}^{\delta(j)}=b_{j} \quad a_{m(j)}^{\delta(j)}=s_{j} x_{j}^{\delta(j)}\left(\left(s_{j} x_{j}^{\delta(j)}\right) \phi\right)^{-1} .
$$

(Note that if $s x((s x) \phi)^{-1} \neq e$, then it is reduced as written [4, Lemma 7.23]. We shall also use the fact that in the product

$$
s x^{\epsilon}\left(\left(s x^{\epsilon}\right) \phi\right)^{-1} \cdot s^{\prime}\left(x^{\prime}\right)^{\delta}\left(\left(s^{\prime}\left(x^{\prime}\right)^{\delta}\right) \phi\right)^{-1} \quad\left(s, s^{\prime} \in S ; x, x^{\prime} \in X\right)
$$

where neither factor is $e$ and the factors are not inverse to each other, neither $x^{\epsilon}$ nor $\left(x^{\prime}\right)^{\delta}(\epsilon, \delta= \pm 1)$ is cancelled in reducing [4, Lemma 7.2.4].) We now distinguish two cases. Firstly suppose $g_{1} u^{n_{j}}$ is an initial segment of $b_{j}^{\prime} s_{j}$; say $g_{1} u^{n_{j}}=b_{j}^{\prime} p_{j}$, where $p_{j}$ is an initial segment of $s_{j}$. Then $\left(g_{1} u^{n_{j}}\right) \phi=p_{j}$.

Secondly suppose $g_{1} u^{n_{j}}$ is not an initial segment of $b_{j}^{\prime} s_{j}$. Thus $g_{1} u^{n_{j}}=$ $b_{j}^{\prime} s_{j} x_{j}^{\delta(j)} q_{j}^{-1}$, where $q_{j}$ is a terminal segment of $\left(s_{j} x_{j}^{\delta(j)}\right) \phi$. Hence

$$
\left(g_{1} u^{n_{j}}\right) \phi=\left(s_{j} x_{j}^{\delta(j)} q_{j}^{-1}\right) \phi=\left(\left(s_{j} x_{j}^{\delta(j)}\right) \phi\right) q_{j}^{-1},
$$

which is an initial segment of $\left(s_{j} x_{j}^{\delta(j)}\right) \phi$.

In either case $\left(g_{1} u^{n}\right) \phi$ occurs as an initial segment of some free generator from $\left\{a_{1}, \ldots, a_{r}\right\}$ or its inverse. However, the elements $g_{1} u^{n_{j}}(j=1,2, \ldots)$ belong to different cosets, since, if $j \neq i$, then

$$
e \neq g_{1} u^{n_{i}} u^{-n_{j}} g_{1}^{-1} \in g U g^{-1} \text {, }
$$


which intersects $H$ trivially. This is impossible since $r$ is finite, i.e. since $H$ is finitely generated.

Remark. It seems likely that (13) can be established for noncyclic malnormal subgroups of $F$.

Note added in proof. The author has found the following more general version of Theorem 2.3, which includes all previous results in the same direction. Its proof requires only slight changes in the above.

Theorem. Let $G=(A * B ; U)$ where there exists a left transversal $T$ for $U$ in $A$, containing $e$, and satisfying

$$
U(T \backslash\{e\})=(T \backslash\{e\}) V_{1}
$$

for some finite subset $V_{1}$ of $U$; and, for every coset $\mathrm{Hg}$ of every finitely generated subgroup $H \leq A$,

$$
H g \subseteq T V_{2}\left(g^{-1} H g \cap U\right),
$$

where $V_{2}$ is a finite subset of $U$ depending on $\mathrm{Hg}$. Then conclusions (i) and (ii) of Theorem 2.3 bold.

\section{REFERENCES}

1. B. Baumslag, Intersections of finitely generated subgroups in free products, J. London Math. Soc. 41 (1966), 673-679. MR 33 \#7396.

2. - Generalized free products whose two-generator subgroups are free, J. London Math. Soc. 43 (1968), 601-606. MR 38 \#2217.

3. L. Greenberg, Discrete groups of motions, Canad. J. Math. 12 (1960), 415-426. MR 22 \#5932.

4. M. Hall, Jr., The theory of groups, Macmillan, New York, $1959 . \quad$ MR 21 \#1996.

5. A. H. M. Hoare, A. Karrass and D. Solitar, Subgroups of finite index of Fuchsian groups, Math. Z. (to appear).

6. A. G. Howson, On the intersection of finitely generated free groups, J. London Math. Soc. 29 (1954), 428-434. MR 16, 444.

7. A. Karrass and D. Solitar, On finitely generated subgroups of a free product, Math. Z. 108 (1969), 285-287. MR $39 \# 6960$.

8. - The subgroups of a free product of two groups with an amalgamated subgroup, Trans. Amer. Math. Soc. 150.(1970), 227-255. MR 41 \#499.

9. W. Magnus, A. Karrass and D. Solitar, Combinatorial group theory: Presentations of groups in terms of generators and relations, Pure and Appl. Math., vol. 13, Interscience, New York, 1966. MR 34 \#7617.

10. D. I. Moldavanskiǐ, The intersection of finitely generated subgroups, Sibirsk. Mat. Ž. 9 (1968), 1422-1426. (Russian) MR 38 \#500.

DEPARTMENT OF MATHEMATICS, YORK UNIVERSITY, TORONTO, ONTARIO, CANADA 